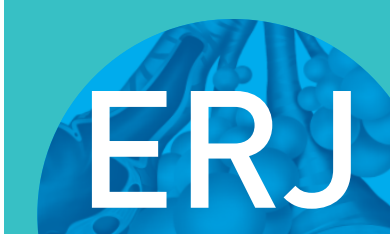

open research

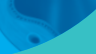

\title{
ERS International Congress, Madrid, 2019: highlights from the Clinical Techniques, Imaging and Endoscopy Assembly
}

\author{
Julia d'Hooghe ${ }^{1}$, Haizea Alvarez Martinez ${ }^{1}$, Pia Iben Pietersen (10,3, \\ Christian B. Laursen (10,4 ${ }^{2,4}$ Nicole Hersch ${ }^{5}$, Ádám Domonkos Tárnoki ${ }^{6,7}$, \\ Simon Walsh ${ }^{8}$, Jouke Annema ${ }^{1}$ and Daniela Gompelmann 9,10
}

Affiliations: ${ }^{1}$ Dept of Respiratory Medicine, Academic Medical Center, University of Amsterdam, Amsterdam, The Netherlands. ${ }^{2}$ Dept of Respiratory Medicine, Odense University Hospital, Odense, Denmark. ${ }^{3}$ Regional Center for Technical Simulation, Region of Southern Denmark, Odense, Denmark. ${ }^{4}$ Dept of Clinical Research, Faculty of Health Sciences, University of Southern Denmark, Odense, Denmark. ${ }^{5}$ Dept of Respiratory Medicine, St George Hospital, Sydney, Australia. ${ }^{6}$ Medical Imaging Centre, Semmelweis University, Budapest, Hungary. ${ }^{7}$ Dept of Radiology, Oncologic Imaging Diagnostic Center, National Institute of Oncology, Budapest, Hungary. ${ }^{8}$ National Heart and Lung Institute, Imperial College, London, UK. ${ }^{9}$ Dept of Pneumology and Critical Care Medicine, Thoraxklink University Heidelberg. Heidelberg, Germany. ${ }^{10}$ Dept of Internal Medicine II, Division of Pulmonology, Medical University of Vienna, Vienna, Austria.

Correspondence: Daniela Gompelmann, Dept of Internal Medicine II, Division of Pulmonology, Medical University of Vienna, Währinger Gürtel 18-20, 1090 Vienna, Austria. E-mail: daniela.gompelmannadirectbox.com

ABSTRACT This manuscript summarises the highlights from Assembly 14, "Clinical techniques, imaging and endoscopy", which were presented at the 2019 European Respiratory Society (ERS) International Congress in Madrid, Spain. Novel diagnostic approaches and innovative therapeutic strategies in patients with lung cancer, interstitial lung disease, obstructive airway disorders and infectious diseases are discussed. The authors from the different Assembly 14 subgroups focus on the key take-home messages given new study results, and place them in the context of current knowledge in these areas.

At the 2019 ERS International Congress, encouraging results of numerous trials in the field of interventional pulmonology, imaging and ultrasound were presented. Key topics included novel diagnostic and therapeutic approaches in patients with lung cancer, interstitial lung disease, obstructive airway disorders and infectious diseases.

@ERSpublications

This article summarises some of the developments in interventional pulmonology, imaging techniques and ultrasound presented at the 2019 \#ERSCongress. Key topics included novel diagnostic and therapeutic approaches in various lung diseases. https://bit.ly/2XYF6Fn

Cite this article as: d'Hooghe J, Alvarez Martinez H, Pietersen PI, et al. ERS International Congress, Madrid, 2019: highlights from the Clinical Techniques, Imaging and Endoscopy Assembly. ERJ Open Res 2020; 6: 00116-2020 [https://doi.org/10.1183/23120541.00116-2020]. 


\section{Group 14.01: Interventional Pulmonology}

\section{Lung cancer}

One of the biggest challenges that interventional pulmonologists face is how to safely diagnose small, peripheral lung nodules. The number of patients with peripheral pulmonary nodules (PPNs) that require tissue confirmation is rapidly increasing with the use of computerised chest tomography (CT) and the implementation of lung cancer screening programmes. Navigation to the target lesion is key. At the ERS International Congress, several different methods to reach PPNs were presented.

One novel technique to sample peripheral nodules is bronchoscopic transparenchymal nodule access (BTPNA) where, at the chosen point of entry, a hole is created in the central airway, then dilated to create a new transbronchial pathway to reach a lung nodule [1]. To identify the point of entry, virtual navigation bronchoscopy (VNB) is used to create a 3D reconstruction of the airways, aligning it with the bronchoscopic image and suggesting the path to follow to reach the target peripheral lesion or the point of entry prior to BTPNA [2]. In a multicentre study, 106 patients underwent VNB or BTPNA or combined methods to obtain biopsy samples [3]. Results showed that the combination of VNB and BTPNA provides safe and effective access to the PPNs in the lungs. However, the data related to BTPNA are still very limited and further prospective trials are required to evaluate the safety and efficacy of this approach.

Another approach to PPNs is the use of radial endobronchial ultrasound probes (r-EBUS), which provide $360^{\circ}$ ultrasound images in the peripheral airways. When introducing the EBUS probe into a peripheral lesion, the snowstorm-like whitish ultrasound image is replaced by a solid mass indicating the location of the lesion. Another navigation system is electromagnetic navigation bronchoscopy (ENB), which combines virtual bronchoscopy imaging with an electromagnetic field and a locatable guide to reach the PPN. ENB has been shown to be a promising bronchoscopy tool for accessing PPNs; however, the overall diagnostic yield remains $\sim 70 \%$, and is even lower in lesions $<2 \mathrm{~cm}$ [4]. At the ERS International Congress, a study was presented that considered whether cone beam CT (CBCT) guidance with live 3D fluoroscopic navigation could be of added value in combination with ENB and r-EBUS. The procedure started with ENB and r-EBUS navigation alone, after which verification of navigation accuracy was performed using CBCT imaging. If CBCT imaging showed an inaccurate position of the catheter, subsequent repositioning and consecutive navigation was performed using $\mathrm{CBCT}$ guidance and live 3D lesion and pathway augmentation on fluoroscopy. The results demonstrated that a small repositioning with $\mathrm{CBCT}$ was needed to obtain 58\% navigation success for nodules with a mean nodule size of $11.5 \mathrm{~mm}$. When combined with live 3D CBCT, this resulted in navigation success of $88 \%$ [5]. This trial demonstrates that $\mathrm{CBCT}$ may be helpful as a guiding tool to reach small lung nodules. However, the availability of CBCT in most institutions is limited and therefore it remains questionable whether this approach will be widely used.

Another important topic discussed at the ERS International Congress 2019 was the use of endobronchial ultrasound-guided transbronchial needle aspiration (EBUS-TBNA), which increasingly represents the first-line approach for mediastinal staging in lung cancer patients [6]. There is a lot of discussion about the needle size and its relation to diagnostic yield. A randomised study in 500 subjects found no significant difference between the $19 \mathrm{G}$ and $21 \mathrm{G}$ needles in a final diagnosis of benign, malignant and non-diagnostic samples $(\mathrm{p}=0.928)$. The sensitivity was $77 \%$ and specificity was $100 \%$ for both needles. Both needles were safe with complication rates of 3.2\% (19G) and 3.6\% (21G) [7]. A new, even smaller histological 25G core needle was evaluated in a small group of patients $(n=20)$, which showed no complications and a sensitivity, specificity and positive predictive value of $90 \%, 100 \%$ and $100 \%$, respectively [8]. Summarising these trials, it seems that the needle size seems to play a less important role in the diagnostic yield of EBUS-TBNA. Combining EBUS-TBNA with elastography images can provide additional information about the lymph nodes. When comparing EBUS-elastography $(\mathrm{n}=215)$ with EBUS $(n=285)$, EBUS-elastography showed a lower number of non-diagnostic samples (1.9\% versus $18 \%$; $\mathrm{p}=0.001)$ and a higher rate of malignant results (54\% versus $12 \% ; \mathrm{p}=0.005)$. Using EBUS-elastography, lymph nodes with a blue pattern had the probability of malignant infiltration of $92 \%$ ( $\mathrm{p}=0.004)$. Furthermore, malignant lymph nodes presented with less colour dispersion, a higher ratio of blue pixels and a higher strain ratio [9].

The performance of EBUS-TBNA is also crucial for the diagnosis of centrally located lung tumours without endobronchial abnormalities $[10,11]$. In one international multicentre analysis presented at the congress, the feasibility and diagnostic yield of EBUS-TBNA for centrally located intrapulmonary tumours were retrospectively evaluated. In 159 patients with centrally located tumours, EBUS-TBNA's feasibility to sample the intrapulmonary lesion was found to be $91 \%$. The diagnostic yield was $86 \%$ and mutational analysis was successful in $86 \%$ of cases [12]. This trial confirms the satisfying results of earlier studies that evaluated the efficacy of EBUS-TBNA for the diagnosis of centrally located tumours [10], but also demonstrates that these EBUS-TBNA samples are mostly suitable for molecular analysis. 
Take-home messages

- Transparenchymal nodule access may present another method to diagnose peripheral pulmonary lesions.

- EBUS-TBNA plays a crucial role in the diagnosis and staging of lung cancer. It is the gold standard in mediastinal staging, where the diagnostic yield is independent of needle size. Furthermore, EBUS-TBNA plays an important role in the diagnosis of centrally located tumours.

\section{Obstructive airway diseases}

In the last decade, several bronchoscopic treatments have been evaluated for patients suffering from severe COPD and asthma. Careful patient selection with the aim of delivering personalised treatment with a high probability of success is a priority.

\section{COPD and emphysema}

In selected COPD patients, endoscopic valve therapy is an established treatment that significantly reduces hyperinflation resulting in clinical improvement [13].

Patients presenting with complete fissures and thus, an absence of collateral ventilation are most likely to benefit. At the ERS International Congress, multiple studies investigated patient and target lobe selection for valve therapy to increase treatment success and prevent inappropriate treatments. Comparing quantitative CT fissure analysis with invasive catheter-based Chartis (Pulmonx Inc., Redwood City, CA, USA) measurement showed an additional benefit with use of the Chartis procedure [14]. For the left major fissure, a fissure completeness of $>95 \%$ on CT had a positive predictive value of $91 \%$, whereas a fissure completeness $\leqslant 83 \%$ had a sensitivity of $100 \%$. For the right major fissure, a fissure completeness of $>95 \%$ had a $74 \%$ positive predictive value, with a sensitivity of $100 \%$ when the fissure completeness was $\leqslant 90 \%$. The results of this trial support the clinical decision on which fissure integrity an additional Chartis measurement is appropriate.

Another trial focused on identifying the best target lobe for valve placement by using the lower lobar ventilation $\left(V^{\prime}\right)$ and perfusion $\left(Q^{\prime}\right)$ quantification to select the target lobe [15]. This was associated with larger improvements in pulmonary function tests (PFTs) compared with a planar $V^{\prime} / Q^{\prime}$ scan, which is reliant upon fissures analysed on CT. The authors also found a significantly improved $V^{\prime} / Q^{\prime}$ quantitation of the contralateral non-targeted upper lobe that was positively associated with improved forced expiratory volume in $1 \mathrm{~s}\left(\mathrm{FEV}_{1}\right)$, though the exact mechanism of this is unclear.

Another technique to potentially improve identification of the optimal lobar target for valve implantation may be endoscopic measurement of the lobar oxygen uptake [16]. In 21 patients, it was shown to be feasible to perform 49 measurements using a customised gas measurement set-up after lobar balloon occlusion. A slower decrease in oxygen uptake was significantly correlated with a higher lobar destruction score and was linked with lower arterial and venous vascular volume within the target lobes. This proves what can be expected from a pathophysiological point of view. However, it still remains unclear whether routine measurement of oxygen uptake will facilitate patient selection or whether CT, which reflects the highest lobar destruction score, is sufficient. Another therapeutic approach to reduce hyperinflation in emphysema patients is bronchoscopic thermal vapour ablation (BTVA). A randomised controlled trial, the STEP-UP (Sequential Segmental Treatment of Emphysema with Upper Lobe Predominance) trial, has already demonstrated the efficacy of this novel approach, which induces an inflammatory reaction in the most emphysematous, destroyed lung segments, thus reducing hyperinflation [17]. A multicentre BTVA post-market registry study presented at the ERS International Congress 2019 showed similar safety data for BTVA compared with the safety profile seen in the STEP-UP trial [18]. COPD exacerbations and pneumonia where the most frequently reported adverse events.

A different mechanism of action in the treatment of COPD is to stop cholinergic hyperactivity. Targeted lung denervation (TLD), a novel endoscopic treatment, aims to target cholinergic hyperactivity by disrupting the parasympathetic pulmonary nerves. A double-blind, randomised, sham-controlled study, AIRFLOW-2, was performed in 82 moderate-to-severe COPD patients to look at 1-year safety data [19]. TLD demonstrated a significantly lower rate of severe COPD exacerbations (TLD 12\% versus sham 32\%; $\mathrm{p}=0.039$ ) and respiratory serious adverse events (TLD 15\% versus sham 37\%; $\mathrm{p}=0.042$ ). Furthermore, the TLD arm had a lower rate of re-hospitalisation (TLD 20\% versus sham 70\%; $\mathrm{p}=0.069$ ). The results of this randomised sham-controlled trial suggest that in future there may be an endoscopic approach for patients with a predominant obstructive ventilatory disorder but only slight-to-moderate hyperinflation. 
The mechanism of action of TLD was further investigated in a sub-study of AIRFLOW-2 [20]. The hypothesis was that by disrupting the pulmonary nerves, neuronal acetylcholine release would be decreased with subsequent reduction in airway inflammation. Surprisingly, in AIRFLOW-2, predefined inflammatory markers were unchanged. However, when the group looked at changes in gene expression on bronchial epithelial brushes taken 3 months after both procedures, TLD-treated patients had evidence of altered gene expression that signals reduction in airways inflammation.

\section{Chronic bronchitis}

A novel treatment for chronic bronchitis is metered cryospray (MCS) to treat airway metaplasia and excessive mucus secretion in chronic bronchitis. Delivering liquid dinitrogen to the airways aims to ablate abnormal epithelium, reduce chronic airway inflammation and promote regeneration of a healthy mucosal lining. In a prospective, single-arm safety and feasibility study in 35 patients with COPD (Global Initiative for Chronic Obstructive Lung Disease (GOLD) stages II-III) and mucus overproduction, MCS was associated with clinically significant improvements in health-related quality of life and 6-min walk distance (by $28 \mathrm{~m}$ ) 9 months after treatment [21]. This result is encouraging; further studies should be performed to evaluate the safety and efficacy of MCS.

\section{Asthma}

Bronchial thermoplasty (BT) is a bronchoscopic treatment for severe uncontrolled asthma. BT targets airway remodelling using radiofrequency energy. Early studies showed that BT reduces exacerbations and improves Asthma Quality of Life Questionnaire (AQLQ) scores [22]. The BT Global Registry collects data on subjects treated with BT in a prospective, multicentre study [23]. This real-world study demonstrates sustained improvement in clinical outcomes and a reduction in asthma medication usage 2 years after BT in 157 subjects. That BT significantly reduces the airway smooth muscle (ASM) has been shown in early, relatively small uncontrolled studies and was confirmed by the results of the randomised controlled TASMA (Targets of Bronchial Thermoplasty in Severe Asthma) trial presented at the ERS International Congress 2019, which showed a reduction from $10 \%$ before BT to $4.6 \%$ after BT $(p=0.01)$ in 27 patients [24]. To create a bigger sample size to investigate airway remodelling responses to BT, data were pooled for 99 patients from seven centres [25]. Again, a reduction in ASM mass in 99 patients occurred 6 weeks after BT ( $16 \%$ before BT to $4.4 \%$ after BT; $\mathrm{p} \leqslant 0.0001)$. The reticular basement membrane thickness reduced within 6 weeks $(6.8 \mu \mathrm{m}$ before BT to $5.8 \mu \mathrm{m}$ after BT; $\mathrm{p}=0.024)$. These effects persisted beyond 1 year after treatment. However, the observed improvements in airway remodelling parameters did not translate to improvements in clinical outcomes (exacerbation rate, AQLQ and lung function).

\section{Take-home messages}

- Endoscopic lung volume reduction is an established treatment. Current studies focus on precise patient selection. Additional Chartis measurement, a decrease of lobar oxygen uptake or attention to lobar perfusion are some of the predictors to be investigated.

- TLD may be an endoscopic approach for patients with a predominant obstructive ventilatory disorder but only slight-to-moderate hyperinflation.

- MCS may be a novel endoscopic treatment that reduces hypersecretion in patients with chronic bronchitis. Further studies are warranted.

\section{Tuberculosis}

The diagnosis of tuberculosis (TB) is challenging and often requires multiple, time-consuming tests with subsequent costs. Pleural TB is one of the most frequent causes of pleural exudates. In patients with exudative pleural effusion and normal lung parenchyma with suspected pleural TB, thoracoscopic pleural biopsy is advised. One trial presented at the ERS International Congress 2019 investigated the use of the new Xpert MTB/RIF assay (Cepheid, Sunnyvale, CA, USA) on thoracoscopic pleural biopsies [26]. In this study, 134 out of 198 patients who underwent thoracoscopic pleural biopsy were diagnosed with TB. The sensitivity of the Xpert MTB/RIF assay was higher compared with biopsy culture (52.2\% versus $41 \%)$ and had the same specificity (100\%). In addition, the Xpert MTB/RIF assay also provides the status of drug resistance, suggesting the potential value of this new technique in the diagnosis and management of pleural TB. Although this trial demonstrates the utility of the Xpert MTB/RIF assay on thoracoscopic pleural biopsies, we would recommend using this assay in addition to biopsy culture, as the culture provides testing for all drug resistances.

Similarly, EBUS-TBNA combined with Xpert MTB/RIF assay was shown to have a positive result in 42 (75\%) out of 56 patients compared with TB culture (52\%) [27]. Nevertheless, it remains important to send 
specimens for culture as well to diagnose other drugs' resistance and detect rifampicin resistance missed by the Xpert MTB/RIF assay.

Take-home message

- The use of the Xpert MTB/RIF on thoracoscopic pleural biopsies or on EBUS-TBNA specimens has an additional value in the diagnosis of TB.

\section{Bronchial fistula}

Bronchial fistula (BF) after pneumonectomy is a serious complication, associated with high mortality. Diagnostic methods to gain information about the size of the BF prior to the placement of an occluder is needed for successful treatment. Novel BF diagnostic methods and treatments were discussed at the ERS International Congress 2019.

In one trial, eight out of 13 patients with a BF following pneumonectomy underwent a BF measurement using a sizing balloon under fluoroscopy, with subsequent successful occluder installation [28]. In the remaining five patients, where BF size was measured with fibrobronchoscopy and CT scans, the occluders chosen needed re-instillation. This suggests that BF measurement with a sizing balloon might be the recommended technique. Although this study result is encouraging, we would like to point out that measurement with a balloon can be quite subjective and there is also the risk of fistula enlargement. Therefore, further studies would be desirable. Bronchopleural fistula (BPF) presents another management challenge. Selective bronchography can provide information about the presence and number of BPF. When retrospectively compared with CT in 27 patients, selective bronchography demonstrated BPF in $81.5 \%$ versus $55.5 \%$ on CT [29]. For BPF management, ventricular septal defect occluders can be used safely and successfully [30].

Take-home message

- Selective bronchography provides to identify BPF.

\section{Interstitial lung diseases}

Transbronchial cryobiopsy (TBCB) for the diagnosis of interstitial lung diseases (ILDs) was again a key topic at the ERS International congress 2019. TBCB is a promising alternative to surgical biopsy and has the advantage of acquiring larger alveolar tissue samples of a higher quality compared with traditional forceps biopsies. Recently, an Australian accuracy study, the COLDICE (Cryobiopsy versus Open Lung biopsy in the Diagnosis of Interstitial Lung disease allianCE) trial) showed high levels of diagnostic agreement between $\mathrm{TBCB}$ and surgical lung biopsy (histopathological agreement was 70.8\%; diagnostic agreement at multidisciplinary discussion was 76.9\%) [31]. The TBCB expert statement suggested a standardised approach for bronchoscopic TBCB and encouraged the need for further randomised studies concerning diagnostic yield, safety and technical aspects [32].

A large study involving 278 patients with ILD investigated the prognostic significance of a multidisciplinary diagnosis of idiopathic pulmonary fibrosis (IPF) versus non-IPF obtained with cryobiopsy [33]. In patients without a typical usual interstitial pneumonia (UIP) pattern on high-resolution CT (HRCT), cryobiopsy was informative in $83 \%$ (IPF in 43\%, non-IPF in 52\%). Results demonstrated a significant prognostic separation between multidisciplinary IPF diagnosis and non-IPF similar to the pathological distinction between UIP and non-UIP cases. This supports the meaningfulness of cryobiopsies in patients with ILD.

To simplify the procedural aspects of TBCB, a prospective study with 84 patients was conducted performing radial EBUS to guide biopsy under conscious sedation without intubation [34]. This was found to be safe and efficient with a diagnostic yield of $79.8 \%$. However, we still recommend to use either an endotracheal tube or a rigid bronchoscope, so that TBCB is performed safely.

One concern in ILD patients is possible concomitant pulmonary hypertension (PH), which may increase the bleeding risk when performing cryobiopsy. It is known that $\mathrm{PH}$ with a pulmonary artery systolic pressure (PASP) $>40 \mathrm{mmHg}$ is a relative contraindication for TBCB due to risk of bleeding and worsening of hypoxaemia. When retrospectively reviewed, 23 out of 150 ILD patients undergoing cryobiopsy were identified as having PH (PASP $>40 \mathrm{mmHg}$ ) [35]. These patients were pre-optimised and cryobiopsy only performed with a PASP $<45 \mathrm{mmHg}$ under general anaesthesia. Compared to patients with PAPS $<40$ $\mathrm{mmHg}$, there was no significant difference in complications such as bleeding, pneumothorax, worsening 
hypoxaemia and mortality. Another relative contraindication is a forced vital capacity (FVC) $<50 \%$ predicted. In a retrospective study including 151 subjects, a higher risk of pneumothorax (27.9 versus $14.4 \%$; $\mathrm{p}<0.05$ ) was found in patients with a FVC $<50 \%$. The risk of bleeding and mortality was no different [36]. This result demonstrates that cryobiopsy in patients with significantly reduced lung function should still be performed with caution after weighing the benefit-risk profile in experienced centres.

Take-home messages

- TBCB is a valuable diagnostic tool for patients with ILD.

- A standardised approach for bronchoscopic TBCB is crucial to reduce risks and complications. Cryobiopsy should only be performed with caution after weighing the benefit-risk profile, particularly in ILD patients with significant reduced lung function or $\mathrm{PH}$.

\section{Group 14.02: Imaging}

ILDs

Multiple papers relating to updates on IPF imaging were presented at the ERS Intrnational Congress 2019.

Magnetic resonance imaging (MRI) has been emerging as an imaging method to assess ILDs. One of the trials presented compared lung T1 weight sequences in patients with IPF and healthy volunteers acquired during inspiration, expiration and tidal breathing. Results revealed that free breathing lung MRI can visualise the changes in both regional and global lung $\mathrm{T} 1$ signals, which might offer a radiation-free assessment of lung scarring [37]. In this trial, the number of patients and healthy subjects was still very low $(n=26)$, so that further trials are needed that evaluate the usefulness of MRI in ILD patients.

More specifically, previous studies have shown that hyperpolarised gas diffusion-weighted (DW) MRI provides a noninvasive, quantitative assessment of microstructural acinar changes in the lung [38]. However, the utility of 129-xenon diffusion imaging has not been studied previously in ILDs; it was evaluated in a prospective, multicentre trial for the first time and presented at the congress. In the study, diffusion restriction quantified by an apparent diffusion coefficient (ADC) provided information about lung microstructure down to the alveolar level, which was then correlated with diffusing capacity of the lung for carbon monoxide, transfer coefficient of the lung for carbon monoxide and CT fibrosis score on hyperpolarised helium DW [39]. The authors demonstrated that ADC correlates with the diffusion capacity; furthermore, the 129Xe DW-MRI could potentially differentiate changes in the airway microstructure in ILD subtypes. Findings on hyperpolarised 129-Xe MR spectroscopy were also presented, where the ratio of 129-Xe uptake in the red blood cells compared with tissue/plasma was shown to be a more sensitive marker for the longitudinal change in IPF and hypersensitivity pneumonitis patients compared with PFTs [40].

To highlight an interesting lecture: a randomised, double-blind, placebo-controlled positron emission tomography (PET) imaging study confirmed target engagement in the lungs of patients with IPF following a single dose of a novel inhaled $\alpha v \beta 6$ integrin inhibitor, an attractive therapeutic target for IPF, which works by modulating the fibrogenic mechanisms in mouse and human lung cells and tissue. Higher levels of $\alpha v \beta 6$ were seen in IPF compared with healthy subjects, where PET was used to demonstrate the target engagement in the lung, which corresponded to fibrotic areas seen on HRCT [41]. This is a promising study result, as it is the first time a target-specific radioligand has been used to assess target engagement in the lung.

Take-home message

- Hyperpolarised gas DW MRI may play a role in ILD, as it potentially differentiates changes in the airway microstructure in ILD subtypes and provides a sensitive marker for the longitudinal change in IPF.

$\mathrm{PH}$

Cardiac MRI (cMRI) metrics are proposed end-points for pulmonary arterial hypertension (PAH) trials. At the congress, the results of the RESPIRE Study were presented, assessing the repeatability and sensitivity to change of right ventricule (RV) analysis methods using cMRI in PAH. In the trial, 44 PAH patients (27 of whom underwent initiation or escalation of therapy) received cMRI at baseline and then two cMRIs within $24 \mathrm{~h}$ at 6-month follow-up. Interobserver, intraobserver and interscan repeatability were assessed and found to be excellent. The RV end-systolic volume (RVESV) and RV ejection fraction (RVEF) were sensitive markers of the change of metrics with PAH therapy [42]. RVESV decreased and RVEF increased significantly following initiation or escalation of therapy. This study demonstrated that cMRI metrics may be used to control and guide therapy in PAH patients. 
Besides MRI, the role of CT pulmonary angiography (CTPA)-derived right atrial area was emphasised. It is known that right atrial dilatation is associated with tricuspid valve regurgitation, which is a significant predictor of long-term mortality in $\mathrm{PAH}$ [43]. One trial from the UK reported that use of a simple CTPA-derived right atrial area measurement correlated well with mean right atrial pressure and can risk stratify patients with PAH [44]. Further studies are warranted to identify optimal CT prognostic thresholds.

Take-home messages

- MRI metrics (RVESV and RVEF) can be used to control and guide therapy in PAH patients.

- CTPA-derived right atrial area correlated well with mean right atrial pressure and thus can risk stratify patients with PAH.

\section{Obstructive sleep apnoea}

CT-derived right-to-left ventricular diameter measurement is a parameter that is used in patients with acute pulmonary embolism to identify those at high risk. New insights on this topic include the findings of a German group, which reported that RV dysfunction might be underestimated in severe obstructive sleep apnoea (OSA) when being evaluated by the RV/left ventricle (LV) diameter ratio assessment on CTPA [45]. In the trial, 197 patients with acute pulmonary embolism underwent CTPA to assess RV dysfunction by calculating the RV/LV diameter ratio. RV dilatation was significantly more frequent in OSA patients compared with subjects with an apnoea-hypopnea index of $<5 \cdot \mathrm{h}^{-1}$. Furthermore, it could be observed that left-sided congestive heart failure $(\mathrm{CHF})$ and signs of acute myocardial injury were significantly more frequent in patients with severe OSA. Thus, importantly, LV dilatation due to coexisting CHF in severe OSA might prevent the RV/LV diameter ratio being $>1$ even in the presence of clinically relevant $\mathrm{RV}$ enlargement.

Take-home message

- CTPA can be used to assess RV dysfunction by calculating the RV/LV diameter ratio in patients with pulmonary embolism. However, it is important to note that in patients with coexisting CHF, CTPA can underestimate an acute right heart dysfunction.

\section{Obstructive airway disease and emphysema}

$\mathrm{CT}$ is an established diagnostic technique to assess the presence, pattern and extent of emphysema. Besides visual assessment, various software platforms are available that provide emphysema quantification by densitometry. A Swedish pilot study reported that visual CT assessment of emphysema is reliable with a satisfactory inter- and intraobserver variability (Krippendorff alpha values ranging 0.80-0.85) [46]. However, when defining emphysema as an LAV950 (\% low attenuation value below 950 Hounsfield Units) of $\geqslant 10 \%$, a matched paired case-control study with 100 emphysema patients and 100 healthy controls, found the sensitivity of detecting emphysema to be only $27 \%$. Thus, the quantitative measurement of LAV950 via the software used in this study was not able to distinguish between patients with or without visually detected mild emphysema [46]. Large-scale studies are needed to evaluate other quantitative emphysema analysis methods with different LAV thresholds and emphysema indices.

Besides quantitative emphysema analysis, CT allows evaluation of airway abnormalities that represent key pathophysiological features in COPD patients. One of the most interesting CT imaging-based modelling studies proved that there are fewer and smaller airways throughout each of the five lobes in COPD subjects compared with controls. This reduction in airways started relatively proximally in generation 5 airways. The reason for this finding remains unclear but may indicate an abnormal remodelling process in the larger airways [47]. These radiological trials allow characterisation of airway abnormalities and assist in our understanding about the pathophysiological processes in COPD and emphysema patients.

Another focus of research is COPD phenotyping, where CT imaging again plays an important role. Alveolar dimensions of quantitative emphysema subtypes (QES) were correlated with an ADC derived from hyperpolarised helium MRI in smokers with COPD and healthy subjects. Apical, diffuse, senile and vanishing lung QES were associated with a rise in mean ADC, suggesting an increase in mean alveolar volume compared with non-emphysematous lung, with particularly large escalations in the apical and vanishing lung QES. This novel QES approach may facilitate personalisation in COPD [48].

One limitation of CT in emphysema patients is its inability to provide functional information. To overcome this limitation, one study evaluated a CT-based full-scale airway network (FAN) flow model, which assesses ventilation in COPD patients [49]. Thereafter, pulmonary tissue density information was extracted from the $\mathrm{CT}$ and, in combination with PFT results, was used for patient-specific modelling. Afterwards, the ventilation 
calculated in the FAN model was compared with the PFT data and the ventilation hyperpolarised Xenon-129 MRI and ventilation single-photon emission CT imaging. The results of this study revealed that the CT-based FAN model is able to provide ventilation images comparable with other functional imaging techniques.

Take-home message

- Quantitative and qualitative CT are increasingly taking a prominent role in the diagnostic process of emphysema and airway remodelling in COPD patients.

\section{Bronchiectasis}

'The authors of one study developed a novel quantitative bronchiectasis scoring technique for CT (BEST-CT), which assessed the sensitivity of CT features in quantifying lung disease in bronchiectasis patients [50]. The scoring techniques include various parameters obtained by volumetric CT scans of patients with bronchiectasis and chronic Pseudomonas infection: consolidation/atelectasis, bronchiectasis with mucus plugging, bronchiectasis without mucus plugging, airway wall thickening, mucus plugging, ground-glass opacities (GGO), emphysema/bullae, healthy airways and healthy parenchyma. This novel quantitative bronchiectasis scoring technique might be helpful for phenotyping and to measure outcomes in clinical trials in patients with bronchiectasis.

Take-home message

- A quantitative BEST-CT supports phenotyping of patients with bronchiectasis.

\section{Acute respiratory failure}

A Dutch paper showed for the first time that confocal laser endomicroscopy (CLE) in patients with acute respiratory failure can be used to study the alveolar compartment for various causes of respiratory failure in mechanically ventilated patients [51]. The authors concluded that CLE imaging has the potential to distinguish between important causes of respiratory failure in critically ill patients in the intensive care unit.

Take-home message

- CLE may help identify the cause of the acute respiratory failure in critically ill patients.

\section{Group 14.03: Ultrasound}

ILDs

Technical developments have made ultrasound available in many institutions. As a result, researchers have and will continue to expand its use as a tool for diagnosis and disease monitoring. In one trial presented at the ERS International Congress 2019, sonographic patterns in IPF patients were correlated with peripheral fibrotic changes on HRCT, and another trial investigated whether ultrasound could be used to predict severity of IPF by using a 12-zone protocol $[52,53]$. Both studies were small, with 16 and 31 patients, respectively, but concludes that sonographic patterns are positively correlated with HRCT; however, thoracic ultrasound could be misleading in cases with concomitant GGO and large cystic honeycomb. Ultrasound may not only be an important tool in the diagnosis and monitoring of ILD but may also be a valuable method for detecting complications following cryobiopsy in ILD patients. One study assessed the diagnostic accuracy of post-procedure ultrasound for diagnosing pneumothorax in ILD patients after TBCB [54]; the authors found a low sensitivity $(20 \%, 95 \%$ CI $2.52-55.6 \%)$ compared to the literature [55], but a high specificity $(100 \%, 95 \%$ CI 95-100). In this setting, ultrasound can identify patients in need of immediate treatment for pneumothorax but fails to identify many patients who develop pneumothorax later on post-procedure.

Take-home message

- Transthoracic ultrasound may present an additional tool in the diagnosis of ILD as the sonographic pattern correlates with fibrotic changes in CT.

\section{Pneumonia}

Thoracic ultrasound for the assessment of pneumonia has been explored for years. It is said to overcome many of the challenges and disadvantages of using conventional chest radiography or thoracic CT, e.g. 
exposure to radiation, inter-rater reliability, and low image quality because of insufficient inspiration or supine one-image radiography. Ultrasound has been proven to have a high sensitivity, specificity and diagnostic accuracy for pneumonia in adults [56-58]. Evidence has been more sparse in the paediatric population. Three studies were presented on this topic at the ERS International Congress 2019.

One trial explored the prognostic role of air bronchograms (static, dynamic, and both static and dynamic in consolidated lung tissue) in the management of children admitted with pneumonia [59]. The authors adopted a grading system called the USINCHILD score. They found that in children with proven community-acquired pneumonia, a difference in USINCHILD score of $<1$ point within the first $48 \mathrm{~h}$ was associated with an increased risk of complicated pneumonia and longer hospitalisation.

In another trial, the authors hypothesised that thoracic ultrasound in children admitted with signs of acute bronchiolitis could predict the need for oxygen therapy [60]. The authors found a statistically significant difference between the ultrasound scores of the group that needed supplementary oxygen therapy and the group that did not need supplementary oxygen therapy.

Souza et al. [61] assessed the inter-operator agreement in thoracic ultrasound for pneumonia in children and achieved very important results. In the study, a novice and an experienced ultrasound operator scanned 23 children with suspected pneumonia. The results showed substantial inter-operator agreement for normal sonographic patterns and lung consolidation ( $\mathrm{K}=0.615$ and $\mathrm{K}=0.635$, respectively), only moderate agreement for B-lines $(\mathrm{K}=0.573)$ but almost perfect agreement for pleural effusion $(\mathrm{K}=0.868)$. The authors concluded that even if scans are performed by operators with very different levels of experience, thoracic ultrasound has a high sensitivity, especially for consolidated lung tissue and pleural effusion.

Take-home message

- Ultrasound has a high sensitivity, specificity and diagnostic accuracy for pneumonia in adults. A number of trials have confirmed the usefulness of ultrasound in children with pneumonia. This is of value as the performance of ultrasound is superior to a chest radiography in children; moreover, radiation exposure is avoided.

\section{Diaphragmatic dysfunction}

One major topic was covered in several studies: assessment of the diaphragm, diaphragmatic function or correlation between diaphragm and peripheral muscle thickness. The diaphragm plays a major role in ventilation as the most important respiratory muscle. Because of this, research to assess its function in chronic, acute and critically ill patients still remains a hot topic.

Lower lung volume due to pleural effusion is not the only factor that causes dyspnoea, as only a very small increase in lung function is seen after thoracocentesis. One trial presented on the ERS International Congress 2019 evaluated diaphragm function prior to and following thoracocentesis in order to explore movement of the diaphragm in dyspnoeic patients with pleural effusion [62]. A midaxillary scan position in conventional B-mode (instead of M-mode, which is most often used for measuring diaphragm function) showed a significant increase in movement after thoracocentesis to a level corresponding to the healthy side.

COPD patients are said to have reduced diaphragmatic movement. One of the studies presented at the congress explored whether it was possible to discriminate between severity in COPD patients (stage I-II and III-IV) by assessing the diaphragm with ultrasound in a stable period without respiratory distress [63]. A statistically significant difference in thickness between the groups during both inspiration and expiration was found, and both measurements correlated with the $\mathrm{FEV}_{1} / \mathrm{FVC}$ ratio. The receiver operating characteristic curve on inspiratory measurement proved that a cut-off-point of $2.63 \mathrm{~mm}$ resulted in a sensitivity of $71.4 \%$ and a specificity of $100 \%$. The authors concluded that ultrasound of the diaphragm could be used to assess severity in certain COPD subtypes, but also acknowledged that other already published studies did not demonstrate a link between COPD severity and diaphragm thickness and movement [64]. This hypothesis needs to be further explored.

Whereas in this trial COPD patients in a stable period were assessed, THABET et al. [65] assessed the correlation between diaphragmatic and peripheral muscle thickness in mechanically ventilated COPD patients. They measured the thickness of the diaphragm and two peripheral muscles on alternate days during mechanical ventilation until the end of mechanical ventilation and found that the change in diaphragmatic thickness differed significantly from changes in peripheral muscle thickness. They concluded that measuring peripheral muscles cannot predict changes in diaphragmatic thickness; however, 
as a secondary outcome, they found that an early decrease in diaphragmatic thickness may be a sign of an extended hospital stay.

Take-home message

- Sonographic assessment of diaphragmatic function and muscle thickness may be useful to the assess severity of COPD. However, the results of various trials are controversial, so further studies are needed.

\section{Peripheral lung lesions}

Ultrasound-guided transthoracic biopsies are also gaining substantial attention because the risk of pneumothorax is lower than CT-guided biopsies in peripheral lesions [66, 67]. Additionally, it is a procedure that can be performed by sufficiently trained pulmonologists, which minimises transfer of patients from one department or institution to another. A first prospective nationwide (French) study evaluated the diagnostic yield and complication rate after ultrasound-guided transthoracic lung biopsies performed by pulmonologists [68]. The results of this trial confirmed the work of previously published studies, [69] noting a high diagnostic yield (85.5\%) and an acceptable complication rate (15\%). The mean procedure time was short at $18.7 \mathrm{~min}$, and the mean pain score was low (1.5 out of 10 on a numerical rating pain scale).

Take-home message

- Ultrasound-guided transthoracic biopsies for diagnosis of peripheral lung lesions have a high diagnostic yield and an acceptable complication rate.

\section{Concluding remarks}

This article summarises only some of the many exciting developments in interventional pulmonology, imaging techniques and ultrasound that were presented at the ERS International Congress 2019. The congress was the first to present in this new format, with its three independent Groups. This new Assembly is strongly committed to providing ERS members with high-quality activities during the congress. In order to do so, member input and feedback is highly desired. We encourage readers to participate in the forthcoming ERS International Congress and to follow-up on their personal topics of interest.

Conflict of interest: J. d'Hooghe has nothing to disclose. H. Alvarez Martinez has nothing to disclose. P.I. Pietersen has nothing to disclose. C.B. Laursen has nothing to disclose. N. Hersch has nothing to disclose. Á.D. Tárnoki has nothing to disclose. S. Walsh has nothing to disclose. J. Annema reports grants from Boston Scientific, Mauna Kea Technologies and COOK Medical, outside the submitted work. D. Gompelmann reports personal fees for lectures and travel fees from Pulmonx, Novartis, Boehringer Ingelheim, Olympus, Chiesi, AstraZeneca, Berlin Chemie and Uptake Medical, outside the submitted work.

\section{References}

1 Herth FJ, Eberhardt R, Sterman D, et al. Bronchsocopic transparenchym nodule access (BTPNA): first in human trial of a novel procedure for sampling solitary pulmonary nodules. Thorax 2015; 70: 326-332.

2 Kemp SV. Navigation bronchoscopy. Respiration 2020; 99: 277-286.

3 Sun J, Vichani A, Criner GJ, et al. Late breaking abstract: Safety and performance of total lung access to peripheral nodules in prospective, multicenter study. Eur Respir J 2019; 54: Suppl. 63, OA1614.

4 Wang Memoli JS, Nietert PJ, Silvestri GA. Meta-analysis of guided bronchoscopy for the evaluation of the pulmonary nodule. Chest 2012; 142: 385-393.

5 Verhoeven R, Fütterer J, Hoefsloot W, et al. Added value of cone beam CT imaging to electromagnetic navigation bronchoscopy in diagnosing small pulmonary lesions. Eur Respir J 2019; 54: Suppl. 63, PA3116.

6 Wahidi MM, Herth F, Yasufuku K, et al. Technical aspects of endobronchial ultrasound-guided transbronchial needle aspiration: CHEST guideline and expert panel report. Chest 2016; 149: 816-835.

7 Tenda ED, Aboelhassan AM, Kontogianni K, et al. Endobronchial ultrasound transbronchial needle aspiration (EBUS-TBNA) versus flexible 19G endobronchial ultrasound transbronchial needle (Flex 19G EBUS-TBNA) in the assesment of mediastinal and hiliar lymphadenophaty: a randomized trial. Eur Respir J 2019; 54: Suppl. 63 , PA324.

8 Gnass M, Rudnicke-Sosin L, Soja J, et al. A usefulness of 25G core needles for immunohistochemistry and molecular testing in lung cancer patients - a feasibility study. Eur Respir J 2019; 54: Suppl. 63, PA4776.

9 Zarogoulidis P, Porpodis K, Xatzibougias D, et al. EBUS elastography for rapid on site lymph node evaluation: a useful tool? Eur Respir J 2019; 54: Suppl. 63, PA3071.

10 Nakajima T, Yasufuku K, Fujiwara T, et al. Endobronchial ultrasound-guided transbronchial needle aspiration for the diagnosis of intrapulmonary lesions. J Thorac Oncol 2008; 3: 985-988.

11 Kuijenhoven JC, Leoncini F, Crombag LC, et al. Endobronchial ultrasound for the diagnosis of centrally located lung tumors: a systematic review and meta-analysis. Respiration 2020; 99: 441-450. 
12 Kuijvenhoven JC, Livi V, Morandi L, et al. The expanding role of endobronchial ultrasound in patients with centrally located intrapulmonary tumors. Eur Respir J 2019; 54: Suppl. 63, PA4772.

13 Herth FJF, Slebos DJ, Criner GJ, et al. Endoscopic lung volume reduction: an expert panel recommendation - update 2019. Respiration 2019; 97: 548-557.

14 Klooster K, Koster DT, Glösenkamp CR, et al. Functional measurement of collateral ventilation is of additional value to CT scan fissure analysis in endobronchial valve treatment for emphysema. Eur Respir J 2019; 54: Suppl. 63, PA3405.

15 Yang L, Hersch N, Ho-Shon K, et al. The value of lobar ventilation and perfusion in selecting targeted lobe for endoscopic lung volume reduction in COPD. Eur Respir J 2019; 54: Suppl. 63, OA5168.

16 Welling J, Klooster K, Charbonnier JP, et al. Feasibility of endoscopic oxygen uptake measurement for target lobe selection in endobronchial valve treatment. Eur Respir J 2019; 54: Suppl. 63, OA5173.

17 Herth FJ, Valipour A, Shah PL, et al. Segmental volume reduction using thermal vapour ablation in patients with severe emphysema: 6-month results of the multicentre, parallel-group, open-label, randomised controlled STEP-UP trial. Lancet Respir Med 2016; 4: 185-193.

18 Valipour A, Herth F, Grah C, et al. Real world safety of bronchoscopic thermal vapor ablation in a multinational registry. Eur Respir J 2019; 54: Suppl. 63, OA5170.

19 Valipour A, Shah P, Herth F, et al. A double-blind, randomized, sham-controlled study of targeted lung denervation in patients with moderate to severe COPD: AIRFLOW-2 one year outcomes. Eur Respir J 2019; 54: Suppl. 63, OA1615.

20 Srikanthan K, Kistemaker L, Slebos DJ, et al. Targeted lung denervation modulates the bronchial epithelial transcriptome in COPD. Eur Respir J 2019; 54: Suppl. 63, OA5169.

21 Garner J, Orton CM, Caneja C, et al. Safety and feasibility of metered cryospray (MCS) for patients with chronic bronchitis in COPD: 9 month results. Eur Respir J 2019; 54: Suppl. 63, OA5172.

22 Castro M, Rubin AS, Laviolette M, et al. Effectiveness and safety of bronchial thermoplasty in the treatment of severe asthma: a multicentre, randomized, double-blind, sham-controlled clinical trial. Am J Respir Crit Care Med 2010; 181: 116-124.

23 Fernandez AT, Herth F, Munoz AM, et al. Bronchial Thermoplasty Global Registry: two year Results. Eur Respir J 2019; 54: Suppl. 63, PA3717.

24 Goorsenberg A, D'Hooghe J, Ten Hacken N, et al. Bronchial thermoplasty induced reduction of airway smooth muscle: results from the randomized controlled TASMA trial. Eur Respir J 2019; 54: Suppl. 63, OA1616.

25 Russell R, Aubier M, Pretolani M, et al. Bronchial thermoplasty leads to rapid and persistent improvements in airway remodelling. Eur Respir J 2019; 54: Suppl. 63, PA3718.

26 Akhter N, Khan K, Chawla D, et al. Comparison between the diagnostic yield of tissue Xpert MTB/Rif assay and culture in pleural tuberculosis patients. Eur Respir J 2019; 54: Suppl. 63, OA1617.

27 Chhajed PN, Vaidya PJ, Mandovra NP, et al. EBUS-TBNA in the rapid microbiological diagnosis of drug resistant tuberculous mediastinal lymphadenopahty. Eur Respir J 2019; 54: Suppl. 63, PA325.

28 Bazhenov A, Motus I, Tsvirenko A, et al. How to measure the size of the bronchial fistula? Eur Respir J 2019; 54 Suppl. 63, OA1618.

29 Largo SJ, De Vega Sanchez B, Vicente CD, et al. Selective bronchography (SB) to confirm bronchopleural fistula (BPF) in patients with persistent air leaks. Eur Respir J 2019; 54: Suppl. 63, PA3388.

30 Jiang J, Guo S, Li Y, et al. Closure of bronchopleural fistula with bronchoscopic placement of ventricular septal defect occluders. Eur Respir J 2019; 54: Suppl. 63, OA1619.

31 Troy LK, Grainge C, Corte TJ, et al. Diagnostic Accuracy of Transbronchial Lung Cryobiopsy for Interstitial Lung Disease Diagnosis (COLDICE): a prospective, comparative study. Lancet Respir Med 2020; 8: 171-181.

32 Hetzel J, Maldonado F, Ravaglia C, et al. Transboronchial cryobiopsies for the diagnosis of diffuse parenchymal lung diseases: expert statement from the Cryobiopsy Working Group on Safety and Utility and a call for standardization of the procedure. Respiration 2018; 95: 188-200.

33 Tomassetti S, Marchi M, Ravaglia C, et al. Prognostic validation of cryobiopsy for the diagnosis of interstitial lung diseases. Eur Respir J 2019; 54: Suppl. 63, PA314.

34 Gnass M, Czyzewski D, Filarecka A, et al. Transbronchial lung cryobiopsy under mini-probe EBUS guidance for interstitial lung diseases - multicenter Polish study. Eur Respir J 2019; 54: Suppl. 63, PA315.

35 Bhat R, Bajaj P, Kalpakam H, et al. Widening the scope of cryobiopsy for ILD- pulmonary hypertension (PH) may not necessarily be a contraindication. Eur Respir J 2019; 54: Suppl. 63, OA1621.

36 Bhat R, Kalpakam H, Bajaj P, et al. Pushing the boundaries in cryobiopsy for ILD - is FVC. Eur Respir J 2019; 54 Suppl. 63, PA328.

37 Saunders L, Eaden J, Bianchi S, et al. Free breathing lung MRI T1 in patients with idiopathic pulmonary fibrosis and healthy volunteers. Eur Respir J 2019; 54: Suppl. 63, PA3162.

38 Chan HF, Weatherley ND, Johns CS, et al. Airway microstructure in idiopathic pulmonary fibrosis: assessment at hyperpolarized ${ }^{3} \mathrm{He}$ diffusion-weighted MRI. Radiology 2019; 291: 223-229.

39 Eaden J, Chan H, Hughes P, et al. Hyperpolarised 129-xenon diffusion-weighted MRI in interstitial lung disease. Eur Respir J 2019; 54: Suppl. 63, PA3157.

40 Eaden J, Hughes P, Collier G, et al. Longitudinal change in hyperpolarised 129-xenon MR spectroscopy in interstitial lung disease. Eur Respir J 2019; 54: Suppl. 63, PA3158.

41 Maher T, Simpson J, Porter J, et al. A PET imaging study to confirm target engagement in the lungs of patients with IPF following a single dose of a novel inhaled avß6 integrin inhibitor. Eur Respir J 2019; 54: Suppl. 63, OA246.

42 Swift A, Cogliano M, Oram C, et al. Repeatability and sensitivity to change of right ventricular analysis methods using cardiac magnetic resonance imaging in PAH: results from the RESPIRE Study. Eur Respir J 2019; 54: Suppl. 63, PA3164.

43 Chen L, Larsen CM, Le RJ, et al. The prognostic significance of tricuspid valve regurgitation in pulmonary arterial hypertension. Clin Respir J 2018; 12: 1572-1580.

44 Dwivedi $\mathrm{K}$, Johns $\mathrm{C}$, Goh ZM, et al. CT pulmonary angiography-derived right atrial area can risk stratify patients with PAH and PH. Eur Respir J 2019; 54: Suppl. 63, PA4813.

45 Berghaus T, Geissenberger F, Konnerth D, et al. Right-to-left ventricular diameter ratio at computed tomographic pulmonary angiography in acute pulmonary embolism and obstructive sleep apnea. Eur Respir J 2019; 54: Suppl 63, OA1932. 
Khalil M, Vikgren J, Cederlund $\mathrm{K}$, et al. Visual assessment versus quantitative densitometry by computed tomography for detection of mild emphysema. Eur Respir J 2019; 54: Suppl. 63, PA4812.

7 Ostridge K, Paas K, Gove K, et al. Fewer and smaller airways in COPD subjects measured by CT imaging. Eur Respir J 2019; 54: Suppl. 63, PA4811.

8 Shen W, Yang J, Sun Y, et al. Apparent diffusion coefficient by $3 \mathrm{He}$ MRI and quantitative emphysema subtypes by CT. Eur Respir J 2019; 54: Suppl. 63, OA1929.

9 Kim M, Doganay O, Matin T, et al. Comparison of the thoracic CT-based computational model with hyperpolarized Xenon-129 MRI and SPECT images to assess pulmonary ventilation in COPD patients. Eur Respir J 2019; 54: Suppl. 63, OA1931.

50 Meerburg J, Dragt O, Van De Corput KM-, et al. Novel quantitative bronchiectasis scoring technique for chest computed tomography: BEST-CT. A study within the iABC project. Eur Respir J 2019; 54: Suppl. 63, PA4817.

51 Mooij-Kalverda KA, Wijmans L, Bos L, et al. Confocal laser endomicroscopy (CLE) in patients with acute respiratory failure on the ICU. Eur Respir J 2019; 54: Suppl. 63, PA3170.

52 Smargiassi A, Inchingolo R, Lombardi F, et al. Possible role of chest ultrasonography for the evaluation of peripheral fibrotic changes in patients affected by IPF. Eur Respir J 2019; 54: Suppl. 63, PA4872.

53 Manolescu D, Tudorache E, Fira-Mladinescu O, et al. Twelve lung zones protocol of ultrasound mapping in idiopathic pulmonary fibrosis. Eur Respir J 2019; 54: Suppl. 63, OA5368.

54 Laursen CB, Pietersen p I, Jacobsen N, et al. Focused lung ultrasound for diagnosing iatrogen pneumothorax after transbronchial lung cryobiopsy. Eur Respir J 2019; 54: Suppl. 63, PA4829.

55 Ebrahimi A, Yousefifard M, Kazemi M, et al. Diagnostic accuracy of chest ultrasonography versus chest radiography for identification of pneumothorax: a systematic review and meta-analysis. Tanaffos 2014; 13: 29-40.

56 Orso D, Guglielmo N, Copetti R. Lung ultrasound in diagnosing pneumonia in the emergency department: a systematic review and meta-analysis. Eur J Emerg Med 2018; 25: 312-321.

57 Long L, Zhao HT, Zhang ZY, et al. Lung ultrasound for the diagnosis of pneumonia in adults: a meta-analysis. Medicine (Baltimore) 2017; 96: e5713.

58 Alzahrani SA, Al-Salamah MA, Al-Madani WH, et al. Systematic review and meta-analysis for the use of ultrasound versus radiology in diagnosing of pneumonia. Crit Ultrasound J 2017; 9: 6.

59 Inchingolo R C, Gerardi RE R, Smargiassi A, et al. Prognostic role of ultrasonographic air bronchogram in management of pneumoniae in children. Eur Respir J 2019; 54: Suppl. 63, PA4824.

60 Cappiello AR, Di Mauro A, Abbondanza N, et al. Lung ultrasound predicts the need of oxygen therapy and longer hospitalization in infants with acute bronchiolitis. Eur Respir J 2019; 54: Suppl. 63, PA1018.

61 Souza T, Nadal J, Peixoto AO, et al. Lung ultrasound in children with pneumonia: inter-operator agreement on specific thoracic regions. Eur Respir J 2019; 54: Suppl. 63, PA4828.

62 Skaarup SH, Lonni S, Quadri F, et al. Ultrasound evaluation of diaphragm function following pleuracentesis. A study on mechanisms of relief of dyspnea. Eur Respir J 2019; 54: Suppl. 63, OA5363.

63 Mikura S, Saraya T, Minami T, et al. Diagnostic utility of diaphragm ultrasound for discriminating severity in COPD patients. Eur Respir J 2019; 54: Suppl. 63, OA5366.

64 Baria MR, Shahgholi L, Sorenson EJ, et al. B-mode ultrasound assessment of diaphragm structure and function in patients with COPD. Chest 2014; 146: 680-685.

65 Thabet D, Ghanem MK, Makhlouf HA, et al. Serial ultrasonographic evaluation of diaphragmatic and peripheral muscle thickness in mechanically ventilated COPD patients. Eur Respir J 2019; 54: Suppl. 63, PA4832.

66 Khosla R, McLean AW, Smith JA. Ultrasound-guided versus computed tomography-scan guided biopsy of pleural-based lung lesions. Lung India 2016; 33: 487-492.

67 Lee MH, Lubner MG, Hinshaw JL, et al. Ultrasound guidance versus CT guidance for peripheral lung biopsy: performance according to lesion size and pleural contact. AJR Am J Roentgenol 2018; 210: W110-W117.

68 Simonneau Y, Ballouhey J, Mangiapan G, et al. Ultrasound-guided lung biopsies performed by pulmonologist: a French prospective study. Eur Respir J 2019; 54: Suppl. 63, PA4833.

69 Laursen CB., Naur TMH, Bodtger U, et al. Ultrasound-guided lung biopsy in the hands of respiratory physicians: diagnostic yield and complications in 215 consecutive patients in 3 centers. J Bronchology Interv Pulmonol 2016 23: $220-228$. 